\title{
Epidemiologia de uma série de tumores primários do sistema nervoso central
}

\author{
Graziella Alebrant Mendes ${ }^{1}$, Bárbara Roberta Ongaratti², \\ Júlia Fernanda Semmelmann Pereira-Lima ${ }^{3}$ \\ Universidade Federal de Ciências da Saúde de Porto Alegre (UFCSPA), Porto Alegre, RS, Brasil.
}

\section{RESUMO}

Objetivo: Realizar um levantamento de dados descritivos em uma série de tumores primários do sistema nervoso central. Métodos: Foram avaliados 106 casos de tumores primários do sistema nervoso central, descrevendo a idade média, os sintomas apresentados, os tipos de tumores mais frequentes e sua localização. Resultados: A idade média foi de 47 anos e 71 casos (67\%) foram do sexo feminino. Observaram-se 39 tipos de tumores primários do sistema nervoso central, sendo os mais frequentes os meningiomas (51 casos, 48\%), seguidos por glioblastomas (19 casos, 18\%). Quanto à localização, 51 casos (48\%) apresentaram-se nas meninges. Cefaleia ocorreu em 52\% dos casos. Conclusões: A faixa etária da amostra é similar a de outros estudos. Observou-se predominância de meningiomas em associação à predominância do sexo feminino, no qual este tumor prevalece. Os sintomas apresentados corroboram com os descritos na literatura.

\section{PALAVRAS-CHAVE}

Epidemiologia, neoplasias do sistema nervoso central, sistema nervoso central.

\section{ABSTRACT}

Epidemiology of a series of primary central nervous system tumors

Objective: To conduct a survey of data descriptive in a series of primary central nervous system tumors. Methods: One hundred and six cases of primary central nervous system tumors were evaluated, describing the mean age, associated symptoms, the most frequent subtype of tumors and their location. Results: The mean age was 47 years and 71 cases (67\%) were female. Thirty nine subtypes of primary central nervous system tumors were observed, and the most frequent subtype was meningioma (51 cases, 48\%), followed by glioblastoma (19 cases, 18\%). Regarding location, 51 cases (48\%) were in the meninges. Headache occurred in $52 \%$ of cases. Conclusions: The mean age of the sample is similar to other studies. Meningiomas prevailed in association with female predominance, where this tumor is prevalent. The symptoms corroborate those described in the literature.

\section{KEYWORDS}

Epidemiology, central nervous system neoplasms, central nervous system.

1 Biomédica. Doutoranda do Programa de Pós-graduação em Patologia da Universidade Federal de Ciências da Saúde de Porto Alegre (UFCSPA), Porto Alegre, RS, Brasil.

2 Biomédica, mestranda do Programa de Pós-graduação em Patologia da UFCSPA, Porto Alegre, RS, Brasil.

3 Médica endocrinologista, doutora em Patologia pela UFCSPA, Porto Alegre, RS, Brasil. 


\section{Introdução}

Os tumores primários do sistema nervoso central (SNC) são um grupo heterogêneo de condições neoplásicas que acometem todas as faixas etárias e podem ocorrer em qualquer ponto anatômico cerebral ou medular. ${ }^{1}$ São considerados a segunda causa mais importante de morte relacionada a doenças neurológicas, sendo superados apenas pelos acidentes vasculares cerebrais. ${ }^{2}$ Nas últimas décadas, observou-se considerável aumento na incidência de tumores do SNC, principalmente em faixas etárias mais avançadas. Isso se deve, em partes, à melhoria nas técnicas de imagem para diagnóstico, como a ressonância magnética, a tomografia computadorizada, entre outros. ${ }^{3}$ A Organização Mundial da Saúde (OMS) propõe uma classificação para esses tumores, de grau I até grau IV, sendo feita a partir da histologia e padrão arquitetural das lesões primárias. Além desse tipo de avaliação, técnicas de imuno-histoquímica, citogenética e biologia molecular também são empregadas para subclassificar esses tumores. ${ }^{4}$ Segundo um levantamento realizado pelo Registro Central de Tumores Cerebrais dos Estados Unidos, entre 2005 e 2009, e que totalizou 311.202 registros, os meningiomas representam o tumor primário de SNC mais frequente $(35,5 \%)$, seguido pelo glioblastoma $(15,8 \%)$. Os sítios mais comuns são as meninges $(35,2 \%)$ e a hipófise $(15,3 \%)$. Os meningiomas representam o tumor de SNC mais comum, comprometendo principalmente adultos acima de 65 anos, sendo incomuns em crianças, e duas vezes mais frequentes no sexo feminino. Os glioblastomas são o segundo tumor mais frequente, representando cerca de $16 \%$ e, da mesma forma que os meningiomas, são mais prevalentes em adultos acima dos 65 anos e raros em crianças. Glioblastomas são 1,6 vez mais comuns em homens. ${ }^{5} \mathrm{O}$ objetivo do estudo foi realizar um levantamento de dados descritivos em uma série de tumores primários do SNC, incluindo a idade, o sexo, o tipo de tumor mais frequente, a localização do tumor e os sintomas apresentados.

\section{Métodos}

Trata-se de um estudo transversal observacional. Foram incluídos os casos de tumores primários do SNC de pacientes submetidos à neurocirurgia no Hospital São José do Complexo Hospitalar Santa Casa de Misericórdia de Porto Alegre entre fevereiro de 2007 e julho de 2010. Foram excluídos pacientes que apresentavam como resultado do exame anatomopatológico tumores hipofisários ou tumores metastáticos de SNC. Os prontuários foram revisados a fim de buscar dados como sexo, idade, localização tumoral, diagnóstico anatomopatológico e sintomas apresentados. A idade foi descrita por média e desvio-padrão e demais variáveis foram avaliadas por frequência e porcentagem. O estudo está condicionado ao cumprimento dos princípios éticos contidos na Declaração de Helsinki, sendo aprovado pelo Comitê de Ética em Pesquisa do Complexo Hospitalar Santa Casa de Misericórdia, parecer 263/09. Todos os participantes assinaram o Termo de Consentimento Livre e Esclarecido (TCLE).

\section{Resultados}

Foram incluídos no estudo 106 casos, cuja idade média foi de 47 anos (DP 15,97), sendo 71 casos (67\%) do sexo feminino. Observaram-se 39 tipos de tumores primários do $\mathrm{SNC}$, sendo os mais frequentes os meningiomas (51 casos, 48\%), entre os quais predominou o tipo meningioma transicional (22 casos, $21 \%$ ), seguido por glioblastomas (19 casos, 18\%), schwannomas (10 casos, 9\%), astrocitomas (5 casos, 5\%) e outros (21 casos, 20\%). Quanto à localização, 51 casos (48\%) apresentaram-se nas meninges, 41 casos (39\%) foram supratentoriais, 12 casos (11\%), infratentoriais e dois casos (2\%) localizaram-se na medula espinhal (Tabela 1). Cefaleia ocorreu em $52 \%$ dos casos, alterações sensoriais, predominantemente visuais, ocorreram em 38\% dos casos e crises convulsivas e náuseas e/ou vômitos ocorreram em $10 \%$ e $13 \%$ dos casos, respectivamente.

\section{Discussão}

A incidência de tumores primários do SNC é de 20,59 para cada 100.000 habitantes. ${ }^{5}$ Segundo estudo epidemiológico descritivo realizado na França, o sítio mais comum dos tumores primários de SNC é a região supratentorial representando $43,62 \%$ dos casos, seguido por inespecífico $(27,43 \%)$, meninges $(16,52 \%)$, intracranial e intraespinhal (5,75\%), infratentorial (4,76\%) e ventricular (1,92\%). ${ }^{6}$ Esses dados não corroboram com os encontrados em nosso estudo em que o sítio mais comum foram as meninges $(48 \%)$, supratentorial (39\%), infratentorial (11\%) e medula espinhal (2\%). Isso pode se dever ao fato de que, no estudo citado, os autores incluíram maior número de pacientes menores de 14 anos, em que os tumores mais frequentes, como os astrocitomas pilocíticos, encontram-se comumente na região supratentorial. ${ }^{5}$ Além disso, obtivemos maior número de meningiomas e, consequentemente, a localização tumoral que predominou foram as meninges. 
Tabela 1 - Tumores primários do sistema nervoso central, conforme tipo histológico, número de casos e localização tumoral

\begin{tabular}{|c|c|c|}
\hline Tipo histológico & Número de casos (n) & $\begin{array}{c}\text { Localização } \\
\text { tumoral } \\
\text { (n) }\end{array}$ \\
\hline
\end{tabular}

\section{Meningiomas}

Transicional

Meningotelial

Fibroso

Fibroblástico

Secretor

Atípico

Psamomatoso

Não classificado

Rabdoide

Anaplásico

Angiomatoso

Microcístico

Gliomas

Glioblastoma

Astrocitoma

pilocítico

Astrocitoma

anaplásico

Oligodendrogliom anaplásico

Oligoastrocitoma

Ependimoma

anaplásico

Glioblastoma de

células

gigantes

Glioblastoma

multiforme

Ganglioma

Glioma maligno/

não classificado

Gangliocitoma

Craniofaringiomas

Adamantinomatoso

Papilar

Outros

Schwannoma

Neurinoma de

Verocay

Cordoma

Cordoma

condroide

Germinoma

Hemangioma

cavernoso

Paraganglioma

gangliocítico

Papiloma do

plexo coroide

$\begin{array}{cc}22(20,75 \%) & \text { Meninges }(22) \\ 8(7,54) & \text { Meninges }(8) \\ 4(3,77 \%) & \text { Meninges }(4) \\ 3(2,83 \%) & \text { Meninges (3) } \\ 3(2,83 \%) & \text { Meninges (3) } \\ 3(2,83 \%) & \text { Meninges }(3) \\ 2(1,88 \%) & \text { Meninges }(2) \\ 2(1,88 \%) & \text { Meninges }(2) \\ 1(0,94 \%) & \text { Meninges }(1) \\ 1(0,94 \%) & \text { Meninges }(1) \\ 1(0,94 \%) & \text { Meninges }(1) \\ 1(0,94 \%) & \text { Meninges }(1)\end{array}$

$17(16,03 \%)$

Supratentorial (17)

3 (2,83\%)

$2(1,88 \%)$

$2(1,88 \%)$

$2(1,88 \%)$

$1(0,94 \%)$

$1(0,94 \%)$

$1(0,94 \%)$

$1(0,94 \%)$

$1(0,94 \%)$

$1(0,94 \%)$

$2(1,88 \%)$

$1(0,94 \%)$

$10(9,43 \%)$

$3(2,83 \%)$

$2(1,88 \%)$

$1(0,94 \%)$

$1(0,94 \%)$

$1(0,94 \%)$

$1(0,94 \%)$

$1(0,94 \%)$

Supratentorial (2)
Supratentorial (2)

Supratentorial (2)

Supratentorial (2)

Supratentorial (2)

Supratentorial (1)

Supratentorial (1)

Supratentorial (1)

Supratentorial (1)

Supratentorial (1)

Supratentorial (1)

Supratentorial (1)

Infratentorial (8)

Infratentorial (2)

Medula espinhal (1)

Supratentorial (1)

Supratentorial (1)

Infratentorial (1)

Supratentorial (1)

Supratentorial (1)
A média de idade foi de 47 anos, mostrando igualdade com outro estudo realizado em Porto Alegre. ${ }^{7} \mathrm{Em}$ estudo de Ostrom et al., a prevalência do sexo feminino também foi constatada, e a observação da prevalência feminina pode ser associada aos hormônios envolvidos no desenvolvimento e no crescimento de tumores cerebrais em mulheres, principalmente os meningiomas, pois estudos mostram que existe a presença de receptores de progesterona nas células desse tipo tumoral. ${ }^{9}$ Em estudo realizado nos Estados Unidos, dois terços dos tumores relatados eram gliomas ou meningiomas $(66,2 \%),{ }^{10}$ dado que coincide com o encontrado no presente estudo, em que gliomas e meningiomas somam $66 \%$ dos casos.

Pacientes diagnosticados com tumores cerebrais geralmente apresentam sintomas neurológicos como cefaleias, convulsões e alterações cognitivas. ${ }^{11}$ Tipicamente a cefaleia é difusa, mas pode indicar o hemisfério cerebral em que se encontra o tumor, geralmente é mais intensa e perceptível pela manhã, podendo ocasionalmente ser unilateral e latejante, confundindo-se dessa forma com um quadro de enxaqueca. ${ }^{12,13}$ Em estudo que analisou 111 casos de tumores cerebrais, foi verificado que $48 \%$ dos pacientes apresentavam cefaleia, ${ }^{12}$ dado similar ao nosso estudo. Dados de literatura mostram que as convulsões atingem aproximadamente $50 \%$ dos pacientes com tumores cerebrais, ${ }^{14}$ entretanto, em nossa amostra, apenas $10 \%$ dos casos tiveram crises convulsivas. Normalmente as crises convulsivas são focais, mas podem causar perda total de consciência. ${ }^{15}$ Segundo Mukand et al. ${ }^{16}$ o déficit mais comum encontrado em 51 casos de pacientes com tumores cerebrais foi a perda cognitiva que ocorreu em $80 \%$ dos casos, seguida por fraqueza (78\%) e alterações visuais (53\%). Náuseas e vômitos, com um exame de imagem anormal ou alteração significativa anterior no padrão de cefaleia, podem ser consequências de um tumor. ${ }^{12}$

\section{Meningiomas}

Meningiomas são tumores que, em sua maioria, possuem caráter benigno e, entre os tumores primários intracranianos, representam cerca de $30 \%$. Podem ser encontrados por toda a superfície externa do cérebro, bem como no interior do sistema ventricular. Possuem crescimento lento e os sintomas neurológicos, como dores de cabeça e convulsões, são causados devido à compressão de estruturas adjacentes. ${ }^{17,18}$ São classificados segundo a OMS de grau I a grau III, sendo o grau I o que inclui os benignos, o II compreende os atípicos e o grau III, os anaplásicos e malignos. ${ }^{4}$ Possuem incidência anual de 7.22 casos a cada 100.000 habitantes. ${ }^{5}$ Entretanto, a incidência pode ser maior, levando em conta que alguns meningiomas podem ser assintomáticos. ${ }^{19} \mathrm{~A}$ exposição à radiação ionizante é um fator de risco bem estabele- 
cido, com intervalo de tempo para o desenvolvimento do tumor de aproximadamente 10 anos. ${ }^{20,21}$ Quanto ao tipo histológico, os dados encontrados corroboram parcialmente com os de Backer-Grøndahl et al.,22 em que o transicional foi o subtipo mais prevalente com $39,8 \%$ dos 196 casos de meningiomas, seguido por atípico (29,1\%), meningotelial (17,3\%), fibroblástico $(7,1 \%)$, angiomatoso $(1,5 \%)$, microcístico, clear cells e anaplásico ( $1 \%$ cada) e psamomatoso e secretor $(0,5 \%$ cada). As variantes encontradas no presente estudo foram meningioma transicional (20,75\%), meningotelial (23,8\%), fibroso (3,77\%), fibroblástico $(2,83 \%)$, secretor $(2,83 \%)$, atípico $(2,83 \%)$, psamomatoso $(1,88 \%)$, rabdoide $(0,94 \%)$, anaplásico $(0,94 \%)$, angiomatoso $(0,94 \%)$, microcístico $(0,94 \%)$.

\section{Glioblastomas}

O glioblastoma é o tipo mais comum de tumor maligno do SNC, com uma média de sobrevivência de 15 meses. ${ }^{23}$ Possui prevalência de 3,19 casos a cada 100.000 habitantes da população em geral e, como a maioria dos tumorais cerebrais, a incidência de glioblastomas cresce conforme a idade da população: de 65 a 74 anos, a incidência é de 13,21 a cada 100.000 habitantes, e de 14,64 a cada 100.000 para a faixa de 75 a 84 anos. ${ }^{5}$ Os glioblastomas são classificados segundo a OMS como grau IV dos gliomas, sendo considerados malignos, com crescimento rápido e alta capacidade de se alastrar pelo tecido cerebral normal, além de serem geralmente resistentes à quimioterapia, sendo letais dentro de $9 \mathrm{a}$ 12 meses. ${ }^{4,24}$ Segundo dados apresentados por Yuile et $a .^{25}$, os principais sintomas evidenciados em pacientes com glioblastomas multiforme são: cefaleia (30,1\%), fraqueza $(20,3 \%)$, perda de memória (15\%), déficit visual $(7,5 \%)$, déficit de fala (6\%) e perda da consciência $(3,8 \%)$. O tratamento escolhido comumente é a cirurgia de ressecção e quimioterapia adjuvante. ${ }^{26}$

\section{Schwannoma}

Os schwannomas, também conhecidos como neurinomas, são tumores originados das bainhas (células de Schwann) de qualquer nervo, são benignos e possuem crescimento lento. ${ }^{27} \mathrm{~A}$ incidência desse tipo tumoral é de 1,7 para cada $100.000 .{ }^{5}$ Entre $25-45 \%$ dos schwannomas se encontram na cabeça ou pescoço. ${ }^{28}$ Segundo a OMS, os schwannomas são considerados grau I. Os sintomas neurológicos relacionados com schwannomas dependem da localização e da origem do tumor, incluindo dores de cabeça, paralisia de nervos cranianos, zumbidos, perda de audição e síndrome de Horner. ${ }^{29} \mathrm{O}$ tratamento para esse tipo tumoral é a ressecção cirúrgica, e várias modalidades vêm sendo introduzidas para preservar a função neurológica dos pacientes. ${ }^{30}$

\section{Astrocitomas}

Os astrocitomas são gliomas que se formam a partir dos astrócitos, células que atuam na sustentação das células nervosas. É o glioma mais comum na infância e representa cerca de $75 \%$ dos gliomas, variando de baixo grau (grau I e II) a alto grau (grau III e IV). ${ }^{31}$ Entre a faixa etária de 0 a 14 anos, possui incidência de 1,66 para cada 100.000. ${ }^{5}$ A distribuição entre os sexos é igual e a localização mais comum é no lobo frontal, seguido por parietal. ${ }^{32}$ Os sintomas causados dependem da localização, da agressividade biológica do tumor e da idade do paciente. Esses sintomas inespecíficos são associados à compressão exercida pelo tumor e incluem dores de cabeça, náuseas e vômitos. Algumas das mudanças comportamentais em crianças incluem alterações de personalidade, irritabilidade, alteração da função psicomotora, apatia e declínio do desempenho escolar. ${ }^{30}$

\section{Conclusão}

Apesar de os resultados deste estudo serem similares a de outros citados na literatura, é de fundamental importância identificar dados epidemiológicos visando ao melhor conhecimento da patologia na população brasileira e, sobretudo, em termos de saúde pública, contribuir para a definição de estratégias de prevenção e diagnóstico eficazes.

\section{Conflitos de interesse}

Os autores declaram não haver conflitos de interesse.

\section{Referências}

1. Burger PC, Scheithauer BW. Tumors of the nervous system. Atlas of tumor pathology. 4th edition. Washington: American Registry of Pathology; 2007.

2. Preston-Martin S, Mack WJ. Neoplasms of the nervous system. In: Schottenfeld D, Fraumeni JF. Cancer epidemiology and prevention. 2nd edition. New York: Oxford University Press; 1996. p. 1231-81.

3. Fisher JL, Schwartzbaum JA, Wrensch M, Wiemels JL. Epidemiology of brain tumors. Neurol Clin. 2007;25(4):86790.

4. Louis DN, Ohgaki H, Wiestler OD, Cavenee WK, Burger PC, Jouvet A, et al. The 2007 WHO classification of tumours of the central nervous system. Acta Neuropathol. 2007;114(2):97-109. 
5. Dolecek TA, Propp JM, Stroup NE, Kruchko C. CBTRUS statistical report: primary brain and central nervous system tumors diagnosed in the United States in 2005-2009. Neuro Oncol. 2012;14(Suppl 5):1-49.

6. Baldi I, Gruber A, Alioum A, Berteaud E, Lebailly P, Huchet A, et al. Descriptive epidemiology of CNS tumors in France: results from the Gironde Registry for the period 2000-2007. Neuro Oncol. 2011;13(12):1370-8.

7. Cambruzzi E, Zettler CG, Pêgas KL, Wanderlei ABS, Junior DK, Duarte MR, et al. Perfil e prevalência dos tumores primários do sistema nervoso central no Grupo Hospitalar Conceição, de Porto Alegre, RS. Rev AMRIGS. 2010;54(1):7-12.

8. Ostrom QT, McCulloh C, Chen Y, Devine K, Wolinsky Y, Davitkov $P$, et al. Family history of cancer in benign brain tumor subtypes versus gliomas. Front Oncol. 2012;2:19.

9. Wigertz A, Lönn S, Mathiesen T, Ahlbom A, Hall P, Feychting M; Swedish Interphone Study Group. Risk of brain tumors associated with exposure to exogenous female sex hormones. Am J Epidemiol. 2006;164(7):629-36.

10. Porter KR, McCarthy BJ, Freels S, Kim Y, Davis FG. Prevalence estimates for primary brain tumors in the United States by age, gender, behavior, and histology. Neuro Oncol. 2010;12(6):520-7.

11. Armstrong TS, Gilbert MR. Glial neoplasms: classification, treatment, and pathways for the future. Oncol Nurs Forum. 1996;23(4):615-25.

12. Forsyth PA, Posner JB. Headaches in patients with brain tumors: a study of 111 patients. Neurology. 1993;43(9):1678-83.

13. DeAngelis LM, Payne R. Lymphomatous meningitis presenting as atypical cluster headache. Pain. 1987;30(2):211-6.

14. Schaller B, Rüegg SJ. Brain tumor and seizures: pathophysiology and its implications for treatment revisited. Epilepsia. 2003;44(9):1223-32.

15. DeAngelis LM. Brain tumors. N Engl J Med. 2001;344(2):11423.

16. Mukand JA, Blackinton DD, Crincoli MG, Lee JJ, Santos $B B$. Incidence of neurologic deficits and rehabilitation of patients with brain tumors. Am J Phys Med Rehabil. 2001;80(5):346-50.

17. Claus EB, Bondy ML, Schildkraut JM, Wiemels JL, Wrensch M, Black PM. Epidemiology of intracranial meningioma. Neurosurgery. 2005;57(6):1088-95.

18. Cea-Soriano L, Wallander MA, García Rodríguez LA. Epidemiology of meningioma in the United Kingdom. Neuroepidemiology. 2012;39(1):27-34.
19. Lusis E, Gutmann DH. Meningioma: an update. Curr Opin Neurol. 2004;17(6):687-92.

20. Wiemels J, Wrensch M, Claus EB. Epidemiology and etiology of meningioma. J Neurooncol. 2010;99(3):307-14.

21. Braganza MZ, Kitahara CM, Berrington de González A, Inskip PD, Johnson KJ, Rajaraman P. lonizing radiation and the risk of brain and central nervous system tumors: a systematic review. Neuro Oncol. 2012;14(11):1316-24.

22. Backer-Grøndahl T, Moen BH, Torp SH. The histopathological spectrum of human meningiomas. Int $\mathrm{J}$ Clin Exp Pathol. 2012;5(3):231-42.

23. Perry J, Okamoto M, Guiou M, Shirai K, Errett A, Chakravarti A. Novel therapies in glioblastoma. Neurol Res Int. 2012;2012:1-4.

24. Louis DN. Molecular pathology of malignant gliomas. Annu Rev Pathol. 2006;1:97-117.

25. Yuile P, Dent O, Cook R, Biggs M, Little N. Survival of glioblastoma patients related to presenting symptoms, brain site and treatment variables. J Clin Neurosci. 2006;13(7):747-51.

26. Scott JN, Rewcastle NB, Brasher PM, Fulton D, MacKinnon $\mathrm{JA}$, Hamilton $\mathrm{M}$, et al. Which glioblastoma multiforme patient will become a long-term survivor? A populationbased study. Ann Neurol. 1999;46(2):183-8.

27. Lin CS, Hsu HS, Tsai $\mathrm{CH}$, Li WY, Huang MH. Gastric schwannoma. J Chin Med Assoc. 2004;67(11):583-6.

28. Yang SW, Lin CY. Schwannoma of the upper lip: case report and literature review. Am J Otolaryngol. 2003;24(5):351-4.

29. Kang GC, Soo KC, Lim DT. Extracranial non-vestibular head and neck schwannomas: a ten-year experience. Ann Acad Med Singapore. 2007;36(4):233-8.

30. Kim SH, Kim NH, Kim KR, Lee JH, Choi HS. Schwannoma in head and neck: preoperative imaging study and intracapsular enucleation for functional nerve preservation. Yonsei Med J. 2010;51(6):938-42.

31. Gupta N, Banerjee A, Haas-Kogan D. Pediatric CNS tumors. 2nd edition. California: Springer; 2010.

32. Grossman SA, Osman M, Hruban R, Piantadosi S. Central nervous system cancers in first-degree relatives and spouses. Cancer Invest. 1999;17(5):299-308.

\section{Endereço para correspondência}

Graziella Alebrant Mendes

Centro de Ciências da Saúde

Campus Universitário Dr. Ulysses Guimarães

Rodovia Municipal Jacob Della Méa, km 5.6, Parada Benito

98020-290 - Cruz Alta, RS, Brasil 